\title{
Assessment of a New High-Performance Small-Animal X-Ray Tomograph
}

\author{
J. J. Vaquero, Senior Member, IEEE, S. Redondo, E. Lage, Student Member, IEEE, \\ M. Abella, Student Member, IEEE, A. Sisniega, G. Tapias, M. L. Soto Montenegro, and \\ M. Desco
}

\begin{abstract}
We have developed a new X-ray cone-beam tomograph for in vivo small-animal imaging using a flat panel detector (CMOS technology with a microcolumnar CsI scintillator plate) and a microfocus $\mathrm{X}$-ray source. The geometrical configuration was designed to achieve a spatial resolution of about $12 \mathrm{lpmm}$ with a field of view appropriate for laboratory rodents. In order to achieve high performance with regard to per-animal screening time and cost, the acquisition software takes advantage of the highest frame rate of the detector and performs on-the-fly corrections on the detector raw data. These corrections include geometrical misalignments, sensor non-uniformities, and defective elements. The resulting image is then converted to attenuation values. We measured detector modulation transfer function (MTF), detector stability, system resolution, quality of the reconstructed tomographic images and radiated dose. The system resolution was measured following the standard test method ASTM E1695-95. For image quality evaluation, we assessed signal-to-noise ratio (SNR) and contrast-to-noise ratio (CNR) as a function of the radiated dose. Dose studies for different imaging protocols were performed by introducing TLD dosimeters in representative organs of euthanized laboratory rats. Noise figure, measured as standard deviation, was $50 \mathrm{HU}$ for a dose of $10 \mathrm{cGy}$. Effective dose with standard research protocols is below $200 \mathrm{mGy}$, confirming that the system is appropriate for in vivo imaging. Maximum spatial resolution achieved was better than $\mathbf{5 0}$ micron. Our experimental results obtained with image quality phantoms as well as with in-vivo studies show that the proposed configuration based on a CMOS flat panel detector and a small micro-focus $X$-ray tube leads to a compact design that provides good image quality and low radiated dose, and it could be used as an add-on for existing PET or SPECT scanners.
\end{abstract}

\section{INTRODUCTION}

$\mathbf{T}$ HE use of nuclear medicine imaging techniques (positron or single photon emission) with small laboratory animals is of increasing relevance in biomedical research. However, these studies are sometimes difficult to interpret due to an ambiguous localization of the tracer uptake. To avoid this problem, registration of PET and SPET images with accurate anatomical images, such as X-ray micro-CT [1] has proven to be an effective means of improving signal localization. Multimodality imaging systems, which are able to carry out combined and intrinsically registered PET-CT and SPET-CT studies, have recently been introduced for humans.

Manuscript received January 15, 2007; revised November 22, 2007. This work was supported in part by the Ministerio de Educación y Ciencia under Grant No. TEC2004-07052-C02, Comunidad de Madrid Grant No. GR/SAL/ 024104, and the CDTEAM project, CENIT program, Ministerio de Industria.

The authors are with the Hospital General Universitario Gregorio Marañón, Madrid 28007, Spain (e-mail: juanjo@mce.hggm.es).

Digital Object Identifier 10.1109/TNS.2008.922814
$\mathrm{X}$-ray micro computed tomography is the preferred anatomical modality for small animal imaging, due to its high-resolution capabilities and to the possibility of integrating it with nuclear medicine imaging devices. Early system implementations made use of detectors based on X-ray image intensifiers and charge-coupled devices (CCD) to which a scintillator screen had been coupled directly or using light guides. Recent developments on semiconductor detectors have made possible the use of new, compact devices for the X-ray detection. A comparison between direct semiconductors (amorphous selenium, a-Se), indirect semiconductor (amorphous silicon, a-Si) coupled to phosphor screens, and CCDs coupled to fiber tapers and phosphor screens, can be found in [2]. In this work the authors conclude that the final CT reconstructed image quality may not be predicted from the detective quantum efficiency (DQE) differences of the detectors studied.

Different micro-CT systems based on a micro-focus X-ray tube and a semiconductor flat-panel detector arranged in conebeam geometry have been developed in the last two decades and its suitability has been proved [3], [4]. The proposed configuration presents some advantages over other alternatives used in clinical and preclinical applications: reduction of acquisition time, large axial field of view (FOV) without geometrical distortions, optimization of radiated dose per time and data acquired, and a more compact, space-saving detector. Additionally, the characteristics of semiconductor flat panels are particularly interesting for small animal imaging due to their high-resolution capability, specially when the micro-columnar scintillator screen is directly grown on the semiconductor detector [5], [6], and current advances in semiconductor technologies suggest an improvement of their characteristics in the future.

We report a new micro-CT scanner devised to be used as an add-on for our previously developed small-animal PET systems [7], [8]. The system was designed to obtain a FOV appropriate for small rodents, a spatial resolution better than 50 micron, and a minimal radiated dose [9]. The tomograph integrates a flat panel (CMOS technology with a columnar CsI scintillator plate) with a pixel size of 50 micron, and a microfocus X-ray source with a nominal focal spot of 35 microns. Both elements are placed in a rotating gantry conforming a cone-beam geometry. The magnification factor of 1.6 was the result of applying the conditions of required final resolution, the $75 \mathrm{~mm}$ diameter, $75 \mathrm{~mm}$ long cylindrical FOV needed to oversize the PET FOV, and the mechanical constrains defined by the system size and the radiation shields.

In this work, the new system characteristics have been evaluated in terms of spatial resolution, image contrast, exposure 


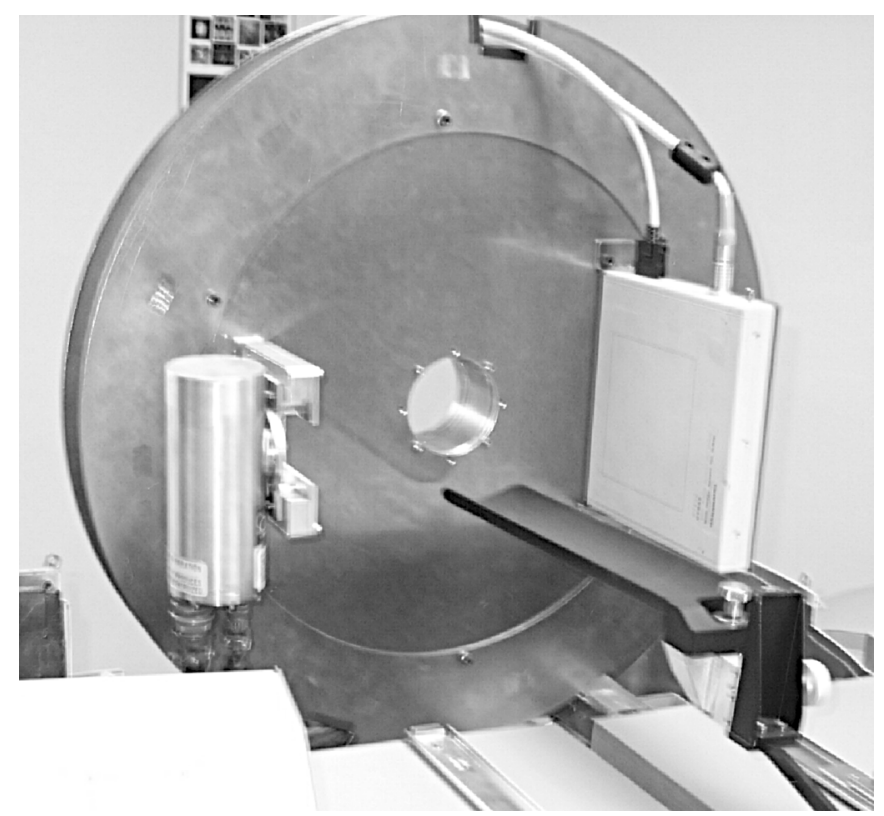

Fig. 1. Small-animal CT prototype showing its components attached to the rotating gantry: micro-focus cone-beam X-ray tube (left), the object bed (center), and CMOS digital imaging sensor (right). Radiation shielding cabinet is not shown.

dose, image acquisition and reconstruction time. We have validated its use for preclinical in-vivo imaging as an add-on system for PET/SPET tomographs or as a standalone unit.

\section{SYSTEM DESCRIPTION}

The scanner design includes a $1.8 \mathrm{GHz}$, dual-core computer that controls a micro-focus X-ray tube (series 5000 Apogee, Oxford Instruments) and a CMOS flat-panel detector, both assembled in a common rotating gantry. A linear motion stage is used to displace the object or animal being scanned along the FOV, thus enabling the tomograph to perform whole body scans (Fig. 1). The assembly is enclosed in a radiation-shielded cabinet with practicable openings for animal positioning, anaesthesia gases lines and physiological signal cables.

The computer controls the system elements during data acquisition to synchronize the gantry motions with the image integration in the detector. The data acquired for each angular position are captured by a digital frame grabber (Epix Inc., Buffalo Grove, IL), which serves as an interface with the acquisition software. Raw data are processed simultaneously with the acquisition, eliminating detector non-uniformities and generating a corrected attenuation image (projection) for each angular position of the gantry.

\section{A. Source and Detector}

The micro-focus X-ray tube has a stationary tungsten anode, a beryllium window of 127 micron thickness, and a Gaussian like focal spot size of $46.5 \mu \mathrm{m} \times 49.1 \mu \mathrm{m}$, as stated in the manufacturer specifications (Fig. 2). Furthermore, the tube has a maximum anode power of $75 \mathrm{~W}$, limited to $50 \mathrm{~W}$ by the high voltage power supply (50 kV for an anode current of $1 \mathrm{~mA}$ ).

The working settings for this component, i.e., the anode current and voltage, are managed from the control computer
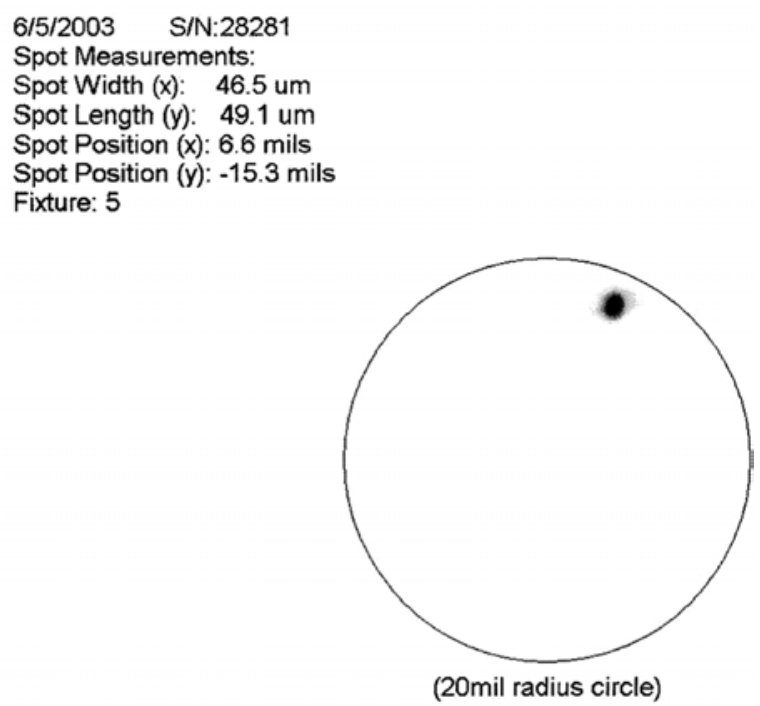

Fig. 2. Focal spot practical measurement provided by the manufacturer (Oxford Instruments) for the tube used in this work.

through an $\mathrm{I} 2 \mathrm{C}$ interface. To reduce the radiated dose during scans, two different elements have been added to the source output window:

- A tungsten shutter used to block the X-ray beam during the intervals in which the detector is not integrating a valid image, i.e., when the gantry is moving. This tungsten shutter is a modified Uniblitz XRS14 with a maximum operating frequency of $10 \mathrm{~Hz}$ and a minimum opening time of $20 \mathrm{~ms}$. The nominal beam extinction ratio goes up to $10^{4}$ for $30 \mathrm{KeV}$ beam maximum energy, as stated by the manufacturer. Practical measures on the fraction of beam extinction for the particular settings of the current system $(40 \mathrm{KeV}, 200 \mu \mathrm{A})$ show a ratio of $4 \cdot 10^{3}$; then, the radiation exposure when the shutter is closed could be considered as negligible.

- An aluminum filter (interchangeable thicknesses from 0.1 to $2 \mathrm{~mm}$ ) is used to filter the low energy region of the emission spectrum, thus improving the beam mono-chromaticity and reducing superficial dose.

No beam collimation is required since most of the X-ray cone bean is used.

The detector is a CMOS flat-panel (Hamamatsu C7942CA) with an active area of $12 \times 12 \mathrm{~cm}^{2}(2400 \times 2400$ pixels, $2240 \times 2344$ effective) and pixel sizes of 200,100 or 50 micron depending on the hardware binning selected. The scintillator is a CsI:TI screen flipped and optically glued to the CMOS. This scintillator is comprised of needle-shaped crystals $0.15 \mathrm{~mm}$ thick grown on a glass substrate. The detector is placed in a plane orthogonal to the X-ray beam axis forming a cone-beam geometry, and it is connected to the control computer through the frame-grabber card that transfers the acquired data to an area of RAM memory accessible for the acquisition software. This configuration makes it possible to achieve transfer rates from 2 up to 9 images per second, depending on the selected binning factor (Table I).

To perform the rotation motion to cover the full angle positions around the scanned item in order to obtain the projection 


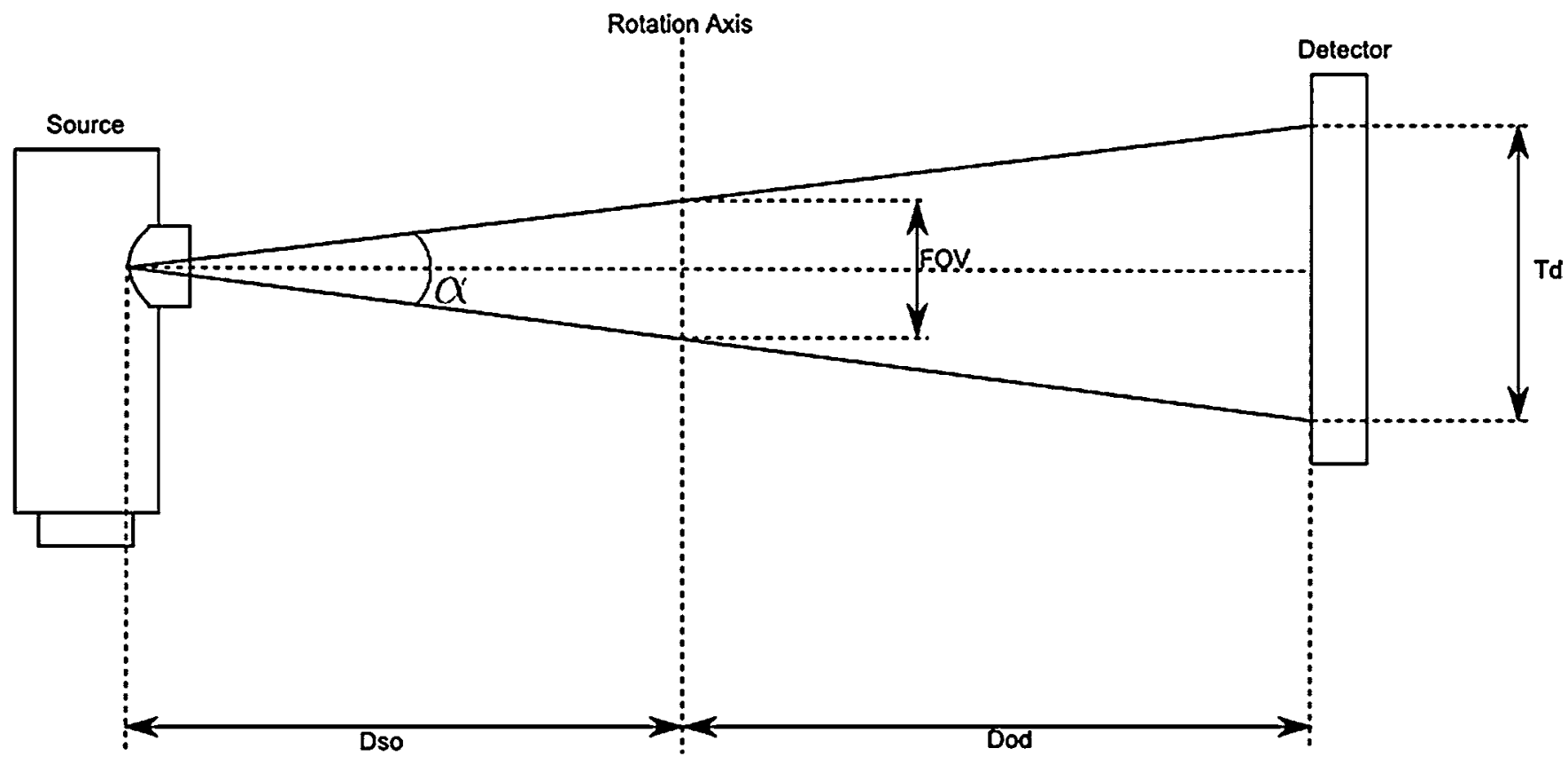

Fig. 3. CT system geometry. $T_{d}$ refers to the usable size of the X-ray CMOS detector.

TABLE I

IMAGE SENSOR FEATURES

\begin{tabular}{ccccc}
\hline $\begin{array}{c}\text { Pixel } \\
\text { Size } \\
(\mu \mathrm{m})\end{array}$ & $\begin{array}{c}\text { Image Size } \\
\text { (pixels) }\end{array}$ & $\begin{array}{c}\text { Utile Area } \\
\text { Size } \\
\text { (pixels) }\end{array}$ & $\begin{array}{c}\text { Data Rate } \\
\text { (images/s) }\end{array}$ & Binning \\
\hline 50 & $2400 \times 2400$ & $2240 \times 2344$ & 2 & 1 \\
100 & $1200 \times 1200$ & $1120 \times 1172$ & 4 & 2 \\
200 & $600 \times 600$ & $560 \times 586$ & 9 & 4 \\
\hline
\end{tabular}

TABLE II

Rotation STAGE FEATURES

\begin{tabular}{cc}
\hline Axis Diameter & $78 \mathrm{~mm}$ \\
\hline Resolution & $0.001^{\circ}$ \\
\hline Accuracy & $0.010^{\circ}$ \\
\hline Repeatability & $0.002^{\circ}$ \\
\hline Maximum Centered Load & $1800 \mathrm{~N}$ \\
\hline Maximum Inertia & $3 \mathrm{~kg} \cdot \mathrm{m}^{2}$ \\
\hline Maximum Speed & $40^{\circ} / \mathrm{sec}$ \\
\hline
\end{tabular}

data to be reconstructed, both source and detector are mounted over a rotation gantry. The motion stage of the gantry is driven by a controller and a stepper motor. The stage is a Newport RV120PP which features are shown in Table II.

\section{B. System Geometry}

The geometrical configuration was designed to accomplish two basic design criteria: the system resolution in the reconstructed image had to be better than 50 micron and the transaxial FOV had to be large enough for small laboratory rodents (about $75 \mathrm{~mm}$ in diameter). The distances from the source to the object $\left(D_{\mathrm{so}}\right)$ and from the object to the detector $\left(\mathrm{D}_{\mathrm{od}}\right)$ were selected accordingly the following expressions [1], [9], [10]:

$$
\mathrm{FOV}=\frac{T_{d}}{M}
$$

$$
M=\frac{D_{\mathrm{so}}+D_{\mathrm{od}}}{D_{\mathrm{so}}}
$$

The useful FOV is defined as the length of the rotation axis intersected by the effective cone-beam (Fig. 3); "effective" refers to the cone-beam section that intersects the active area of the detector. Since the FOV size ( $75 \mathrm{~mm}$ diameter by $75 \mathrm{~mm}$ length) and the resolution were design specifications, and the physical size of the system had to be constrained to the available space, the geometrical adopted solution $\left(\mathrm{D}_{\mathrm{so}}=219.8 \mathrm{~mm}\right.$ and $\mathrm{D}_{\text {od }}=131.9 \mathrm{~mm}, \alpha=19.4^{\circ}, \mathrm{T}_{\mathrm{d}}=120 \mathrm{~mm}$ ) provides a magnification factor (M) of 1.6 (Fig. 3). The theoretical system resolution is calculated as the convolution of the effects of the finite focal spot size in the source $\left(\rho_{\mathrm{f}}\right)$ and the intrinsic resolution of the detector $\left(\rho_{\mathrm{d}}\right)[10]$.

The component of the system resolution due to the detector $\left(\theta_{\mathrm{d}}\right)$, can be calculated in the center of the FOV using the following expression:

$$
\theta_{d}=\frac{\rho_{d}}{M}
$$

If $\rho_{f}$ is the focal spot size of the source, then the $\theta_{f}$ at the center of the FOV is:

$$
\theta_{f}=\rho_{f} \frac{M-1}{M}
$$

Assuming Gaussian distributions for $\theta_{f}$ and $\theta_{d}$, the resulting resolution for the reconstructed images can be estimated theoretically according to the formula:

$$
\theta=\sqrt{\theta_{f}^{2}+\theta_{d}^{2}}
$$




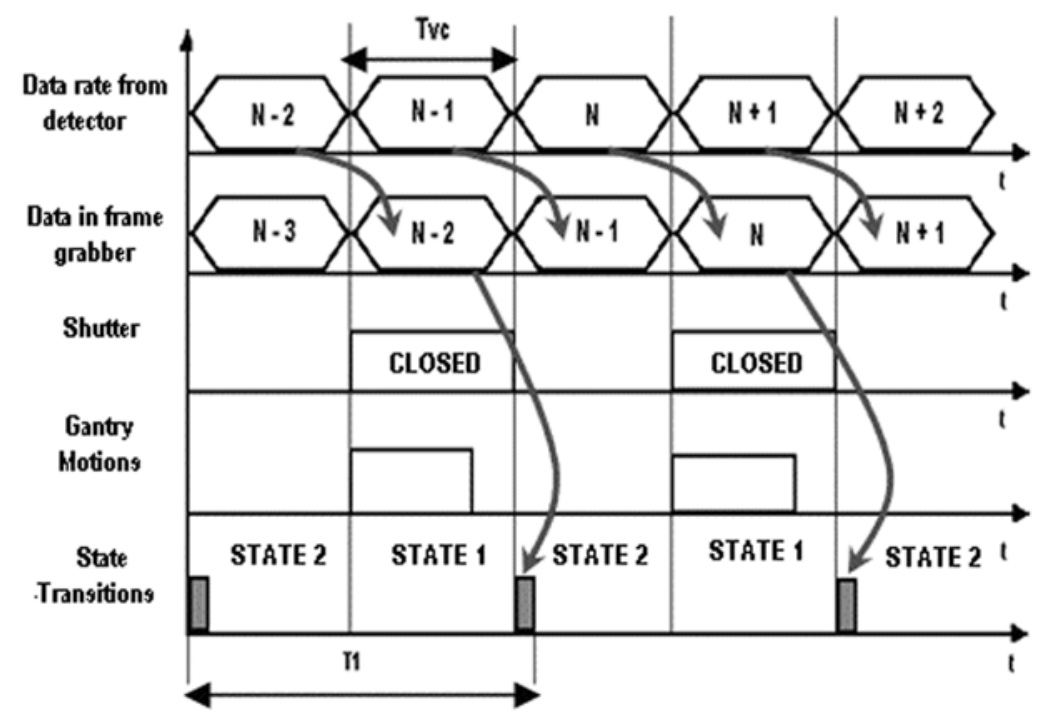

Fig. 4. High-speed acquisition protocol. $\mathrm{T}_{\mathrm{vc}}$ represents the integration period in the detector and $\mathrm{T} 1$ indicates the time between the acquisition start of an image and the storage of already processed images in RAM before they can be saved to disk. The state transitions are shown in the last row of the chronogram and the gray rectangle indicates the time spent by the acquisition software processing the data set acquired in the previous gantry position.

The theoretical resolution for the configuration previously specified is 12 cycles/mm (MTF $10 \%$ ) or about 40 micron in the spatial domain. However, there are additional factors that degrade system resolution derived from the tomographic reconstruction process and submillimeter mechanical misalignments. The alignment between the source and the detector is critical for achieving the specified resolution value, and in this system it is attained by using an analytical procedure based on the assessment of the elliptical trajectories of two ball bearings, as described in [11]. Using the mentioned method we are able to estimate the values of two of the three tilt angles of the detector and the position of the real center for the image (i.e., the projection pixel for the $\mathrm{X}$-ray beam central, orthogonal ray). The remaining angle, although it is not as critical as the previous two, [11]-[13] was previously reduced as much as possible in order to be able to assume that its value is zero. The correct alignment in the direction of the non-estimable angle is mechanically assessed. After the estimation of the different parameters, the tilt angles are mechanically corrected in order to avoid rotation corrections that limit the system accuracy, and the center misalignment is estimated again and corrected by the acquisition software.

\section{Acquisition Protocol}

To take advantage of the maximum detector transfer rate [14], the acquisition protocol was implemented as an event-driven finite-state machine with two possible states. The first one (step) is used to move the gantry to the following angular position and, if needed, to save the data from RAM memory to disk. The second state (shoot) performs two tasks:

a) Launching the acquisition of either a single frame or a frame sequence in the current angular position and

b) Processing the data set acquired in the previous gantry position.

The transition between states is triggered in synchrony with the detector integration period $\left(\mathrm{T}_{\mathrm{vc}}\right)$ as shown in Fig. 4.
The processing carried out by the software to generate a projection image includes the correction of geometrical misalignments between source and detector, correction of defective lines and pixels of the detector using interpolation with the nearest pixels and the conversion of the resulting data (values proportional to the incident photon flux) into attenuation images [15]. This latter step also implements dark current correction and sensitivity normalization using a flood-field image reference. The flood-field image is previously acquired with the X-ray source settings adjusted for the current study and without the object between source and detector.

It is also possible to improve the quality of the projection images by acquiring and averaging more than one frame (from 2 up to 32) at each angular position. In this case, the finite-state machine waits in shoot state for a time equal to $\mathrm{T}_{\mathrm{VC}} * \mathrm{~N}$, where $\mathrm{N}$ is the number of frames, until the last frame for the current projection arrives. Therefore, in the multiframe case there are several "state 2" periods between two "state 1" periods in which the CPU is idling. Additional pre-reconstruction processing, such us filtering, can be done during those intervals.

The acquisition time (in seconds) is given by

$$
T_{\mathrm{acq}}=\left[\frac{1}{\mathrm{FR}}\right] \cdot\left[A v_{\mathrm{img}}+N_{\mathrm{loss}}\right] \cdot N_{p} \cdot N_{\mathrm{FOV}}
$$

where

- FR is the frame rate from the detector, in images per second.

- $A v_{\mathrm{img}}$ is the number of averaged frames per projection image.

- $N_{\text {loss }}$ is the number of frames lost due to the motion of the rotating stage (always 1 in the current implementation).

- $N_{p}$ is the number of views acquired over a $360^{\circ}$ gantry rotation span.

- $N_{\mathrm{FOV}}$ is the number of axial bed positions 
These parameters can be adjusted to configure different acquisition protocols: for example, one option could be a highspeed, low-resolution and low-dose scan, or alternatively a highresolution, high-dose and slower scan. In every case, the exposition time is controlled by synchronizing the gantry rotation and shutter with the frame grabber timing.

\section{IMAGE RECONSTRUCTION}

Images were reconstructed using a modified Feldkamp algorithm [16] with a Ram-Lak filter. The algorithm was adapted to the specific geometry of our tomograph, and includes beam hardening correction and calibration in Hounsfield Units (HU), using a phantom with seven different known materials to fit the acquired data. The calibration is done for four standard settings and interpolation is used for intermediate values. Symmetries are exploited to accelerate the algorithm, and fast back-projection techniques can also be used for low-resolution studies.

\section{ASSESSMENT}

\section{A. CMOS Detector Evaluation}

The detector performance was evaluated in terms of stability and Modulation Transfer Function (MTF): non-stability affects the tomographic images by increasing noise and artefacts introduced by the detector, whereas MTF represents the intrinsic resolution of the detector and limits the final resolution that the system can achieve, as shown in (3)-(5).

To test the detector stability, 360 consecutive flood-field images without any object between source and detector were acquired with the X-rays source set to $30 \mathrm{kV}$ and $0.4 \mu \mathrm{A}$. Each image was acquired after waiting ten seconds to stabilize the source photon flux. The mean pixel value was measured on each raw image. High-voltage was set to zero for ten seconds between acquisitions to extinguish the $\mathrm{X}$-ray flux and let the scintillation decay in order to remove any potential afterglow contamination on the next measurement.

The detector MTF was calculated by direct analysis of the Edge Response Function (ERF). The ERF was obtained from the image of an X-ray opaque object with a polished edge, adjusting its profile to an integrated Gaussian function [17]. The Point Spread Function (PSF) was then calculated as the analytical first derivative of the ERF. The MTF was obtained by taking the module of the Fourier transform of the PSF. The same method was followed to obtain the detector intrinsic MTF, by placing the object close to the detector (no magnification). The composed MTF that reflects the combined effect of the detector and the finite focal spot size of the source was obtained by imaging the object at nominal system magnification [9]. Detective quantum efficiency (DQE) for this type of detectors has been previously evaluated in [4] and [18].

\section{B. Quality Evaluation of Reconstructed Images}

The quality of the reconstructed images determines their utility in preclinical studies. However, longitudinal in vivo

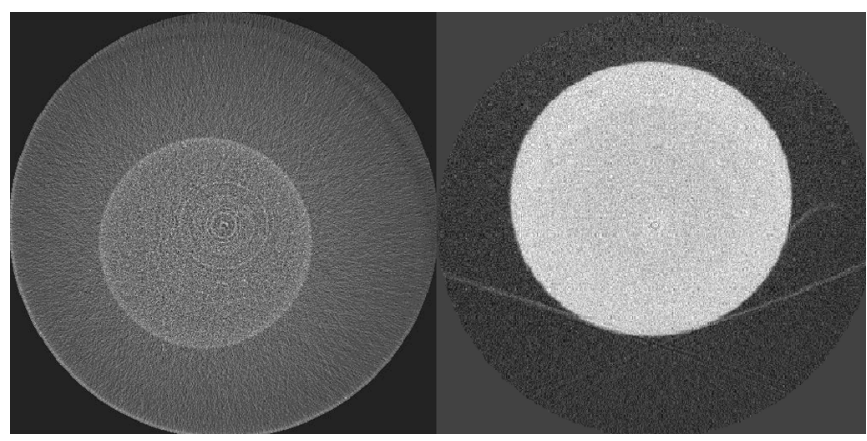

Fig. 5. Transaxial image of the nylon-water phantom used to measure the CNR acquired with $5.3 \mathrm{cGy}$ (left); transaxial image of the polycarbonate disk used to evaluate spatial resolution (right).

studies preclude the use of high doses in the scan; therefore a compromise between dose and image quality must be reached.

The image quality evaluation was based on measuring noise level, Contrast-to-Noise Ratio (CNR) and spatial resolution, which constitute the main features in terms of image quality for preclinical applications.

Noise in reconstructed images was evaluated on a homogeneous water phantom by measuring the standard deviation of the signal in $\mathrm{HU}$, as a function of radiated dose. The phantom was acquired six times at $25 \mathrm{kV}$ and $0.6 \mu \mathrm{A}$ at different doses (different number of averaged images for each angular position).

CNR was measured as a function of radiated dose using a contrast phantom which consists of a nylon cylinder $\left(1.15 \mathrm{~g} / \mathrm{cm}^{3}\right)$ immersed in a water container (Fig. 5). CNR is defined as:

$$
\mathrm{CNR}=\frac{\left|\mu_{n}-\mu_{w}\right|}{\sqrt{\sigma_{n}^{2}+\sigma_{w}^{2}}}
$$

where $\mu$ and $\sigma$ are, respectively, the mean and standard deviation of the pixel values from the reconstructed images in a given area corresponding to the water $\left(\mu_{\mathrm{w}}, \sigma_{\mathrm{w}}\right)$ and the nylon $\left(\mu_{\mathrm{n}}, \sigma_{\mathrm{n}}\right)$. Regions of interest used for the analysis were obtained by grey level thresholding.

The system resolution was measured following the standard test method E1695-95 [19]. This method is based on the examination of the CT image of a uniform disk of polycarbonate $\left(1.18 \mathrm{gr} / \mathrm{cm}^{3}\right)$ (Fig. 5). The resolution measurement is derived from an analysis of the edge of the disk: the ERF is obtained, and the PSF and MTF are calculated. The cut-off point where the MTF decreases to $10 \%$ of its maximum value is given as the standard resolution measurement.

\section{Radiation Dose Evaluation}

To obtain a more precise assessment of biological effects in in-vivo studies than that offered by purely physical exposure measurements, we performed a study to estimate radiation dose. Thermo-luminescent dosimeters (STI, TLD-100) were introduced into representative tissues in euthanized rats before undergoing standard acquisition protocols. 


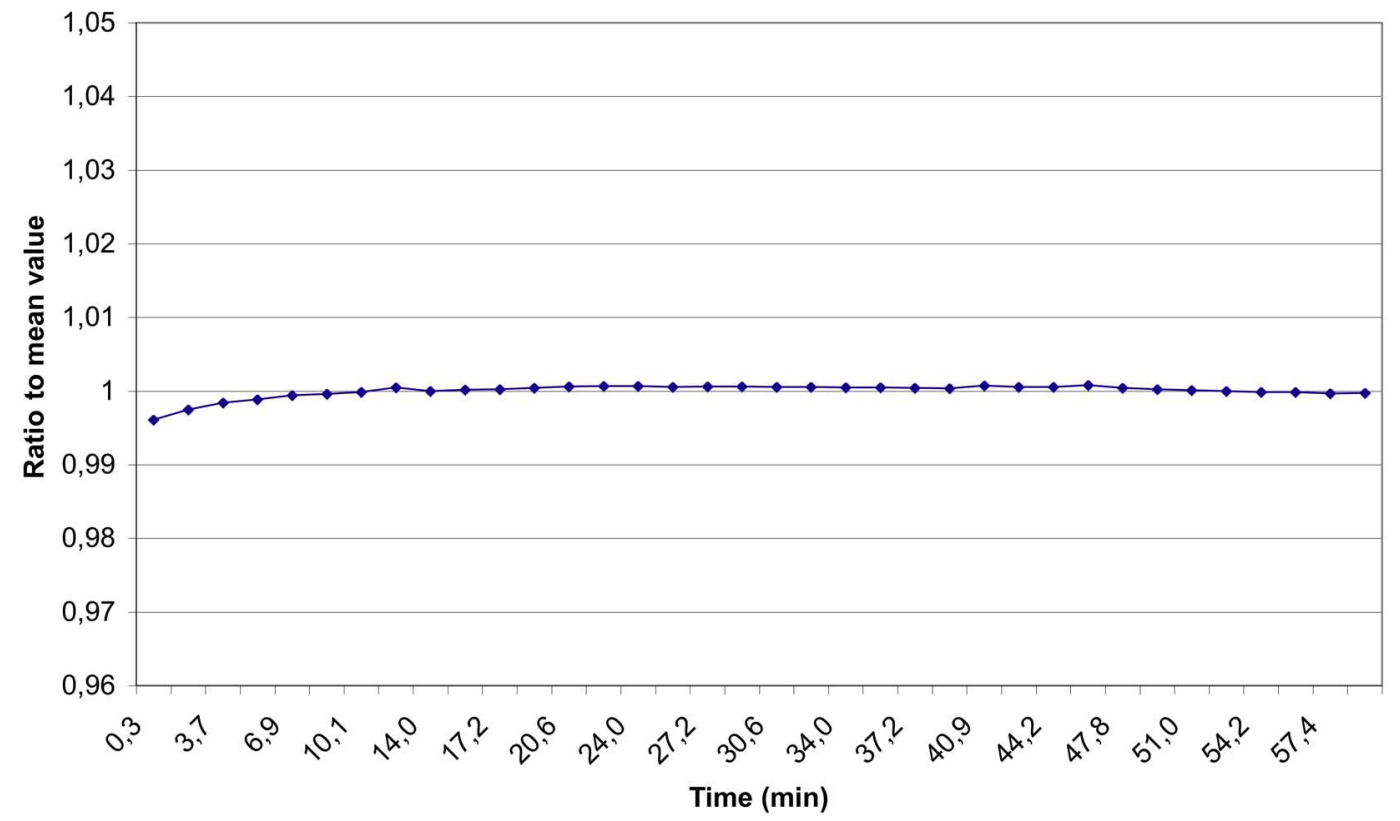

Fig. 6. Detector gain variation as a function of time; mean pixel value normalized to the mean value using every acquired frame. First measurement was delayed ten seconds to allow X-ray flux stabilization.

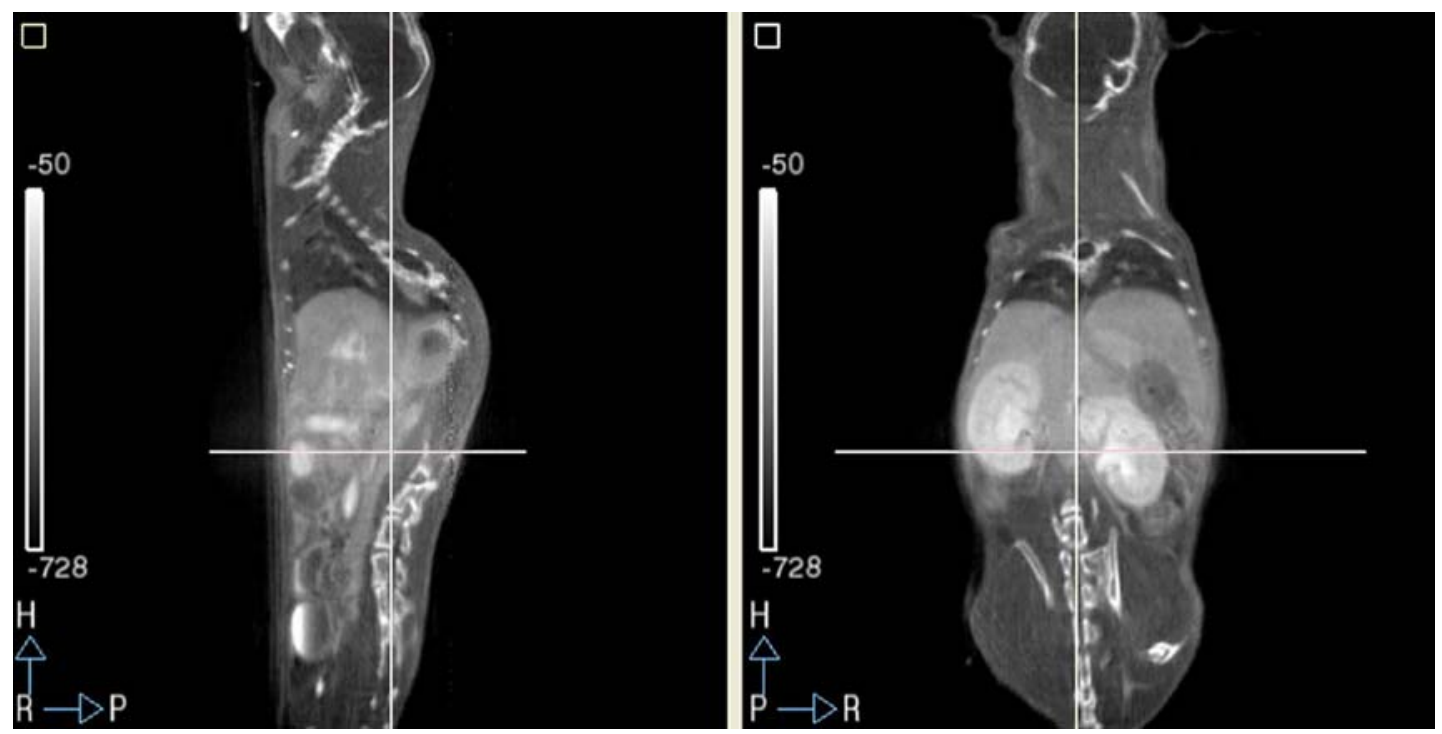

Fig. 7. Sagittal, coronal and axial views of a mouse abdomen. The acquisition parameters were $35 \mathrm{kV}, 200 \mu \mathrm{A}, 360$ angular projections, binning four, eight images averaged per projection (standard lab protocol for mice). Total acquisition time was 400 seconds. The image reconstruction took 90 seconds on a standard personal computer.

\section{RESULTS}

\section{A. CMOS Detector Evaluation}

Detector stability is shown in Fig. 5. This result indicates that the CMOS detector is stable enough, thus the reconstructed images will not be influenced by this parameter.

Intrinsic resolution of the detector, defined as MTF $10 \%$, resulted in $8.1 \mathrm{lpmm}$, a figure compatible with the manufacturer's specifications. MTF 10\% measured at the nominal system magnification was 11.85 cycles per mm, almost 1.6 times the intrinsic resolution.

\section{B. Quality Evaluation of Reconstructed Images}

The optimized reconstruction software and the acquisition real-time pre-processing achieved reasonable reconstruction times on standard personal computers (100 seconds to reconstruct a $512^{3}$ voxels volume using a $2.80 \mathrm{GHz}$ dual core CPU with 8 GB of RAM).

Measured noise level decreased proportionally to square root of dose, as expected according to theoretical noise models for CT images [20]. A good soft tissue contrast is achieved for a noise level below $50 \mathrm{HU}$, corresponding to a radiated dose of $7.5 \mathrm{cGy}$. Fig. 8 shows the curve of noise level as a function of dose.

Fig. 9 shows a plot of the CNR with respect to radiated dose. It can be noticed how CNR increases almost proportionally to the square root of the dose. CNR obtained for a dose of $7.5 \mathrm{cGy}$ is 0.98 .

MTF 10\% measured according to the standard protocol E1695-95 was 11.3 cycles $/ \mathrm{mm}$ or $44 \mu \mathrm{m}$ in the spatial domain. 


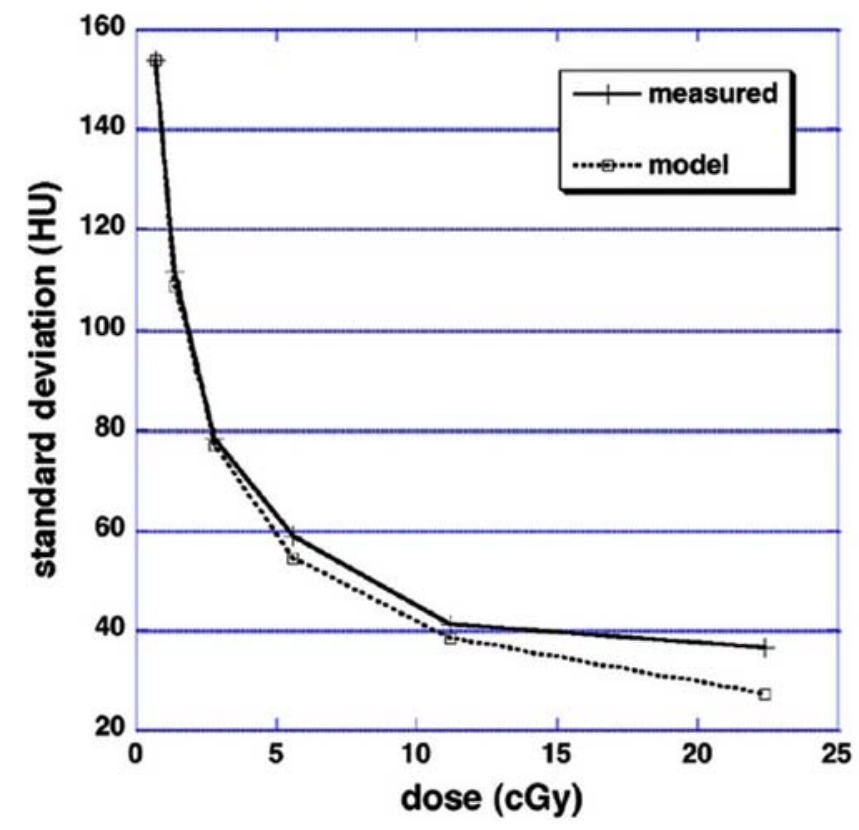

Fig. 8. Noise level (standard deviation) in Hounsfield Units as a function of radiated dose (solid: measured data, dotted: Gaussian model).



Fig. 9. CNR as a function of radiated dose.

The observed actual resolution on the reconstructed images was lower than the resolution measured in projection due to the reconstruction process and to possible submillimeter misalignments [11].

\section{Radiation Dose Evaluation}

Table III shows the results and the acquisition settings for two standard protocols. The first one intends to provide anatomical information for PET-CT studies, in which high resolution is not required because PET image resolution is usually worse than $1 \mathrm{~mm}$. The second protocol represents a high resolution setting for bone tissue, which needs higher voltage and higher current.
TABLE III

X-RAY SetTINGS AND RADIATION DOSE FOR Two STANDARD ACQUISITION PROTOCOLS

\begin{tabular}{ccccc}
\hline \hline Voltage & Current & Time & Resol. & $\begin{array}{c}\text { Radiation } \\
\text { dose }\end{array}$ \\
\hline $25 \mathrm{kV}$ & $600 \mu \mathrm{A}$ & $6^{\prime} 00^{\prime \prime}$ & $200 \mu \mathrm{m}$ & $33,73 \mathrm{mGy}$ \\
$40 \mathrm{kV}$ & $750 \mu \mathrm{A}$ & $6^{\prime} 30^{\prime \prime}$ & $100 \mu \mathrm{m}$ & $165,2 \mathrm{mGy}$ \\
\hline \hline
\end{tabular}

The radiation doses obtained were, respectively, $0.5 \%$ and $2 \%$ of LD50/30 ( 7.3 Gy) for small rodents [21], [22]

\section{DisCUSSION AND CONCLUSION}

We have developed and evaluated a new, compact micro-CT system usable as an add-on for our previously developed smallanimal PET [7], [8]. The system is self-contained and can be easily operated as an independent imaging instrument.

When using low power X-ray sources, one possible way to improve the SNR and therefore the contrast of the images in soft tissue, is to optimize the exposure time. In this context, the optimization of the acquisition protocol allows us to better exploit the detector features, thus improving the system performance with regards to per-animal screening time.

Regarding the choice of the detector, the size of commercial CMOS flat panels makes them suitable for small animal imaging, since they lead to a more compact design than that obtained with CCD detectors, the most common choice in this kinds of systems. We have shown that CMOS flat-panel detectors offer good results in terms of noise, contrast and resolution. These features make it possible to optimize image quality in terms of radiated dose during the study.

With regard to the resolution, the elements and the configuration of the system yield a spatial resolution better than $50 \mu \mathrm{m}$, fulfilling one of the basic design criteria. We have characterized source and detector components of the system resolution and how it is degraded during the image reconstruction process. Imaging of in vivo animals may show a slight quality degradation when operating the system with the maximum resolution protocol due to movements like breathing or heart beating; gating the projections acquisition or doing a retrospective gating over multiple expositions per projection have been used to recover this resolution loss at the cost of an increased radiated dose to the animal. Besides these considerations, we want to stress that high-resolution imaging requires a precise characterization of the system alignment; mechanical misalignments that could not be corrected have to be taken into account during the image reconstruction process, and this may prevent from using symmetries, thus increasing the reconstruction time noticeably.

Considering (3)-(5), which describe how the detector and source components affect the final resolution, the limiting factor in this configuration is the intrinsic resolution of the detector, as the effect of the finite focal spot is less significant. We must consider that under our geometrical constraints and specification requirements, the magnification factor is fixed by the FOV, the cone-beam angle and the physical size of the detector. Therefore, the final resolution would benefit from using new versions 
of the flat-panel detector with improved intrinsic resolution (already available), and no other change to the system.

Results of dosimetry show that the system is suitable for in vivo imaging, especially when using relatively low resolution protocols $(200 \mu \mathrm{m})$. If the resolution is doubled for a given $\mathrm{X}$-ray setting, voxel noise increases four times and the dose has to increase 16 times to maintaining the same SNR [21]. For this reason, special care must be taken with X-ray settings for ultra-high resolution protocols.

\section{ACKNOWLEDGMENT}

The authors thank SUINSA Medical Systems for their support with the system mechanical development, and Prof. C. Antoranz from the Physics Faculty of the UNED for access to his X-ray tomograph system.

\section{REFERENCES}

[1] M. J. Paulus, "High resolution x-ray computed tomography: An emerging tool for small animal cancer research," Neoplasia, vol. 2, pp. 62-70, 2000.

[2] A. L. Goertzen, V. Nagarkar, R. A. Street, M. J. Paulus, J. M. Boone, and S. R. Cherry, "A comparison of x-ray detectors for mouse CT imaging," Phys. Med. Biol., vol. 49, pp. 5251-5265, 2004.

[3] D. A. Jaffray and J. H. Sierwerdsen, "Cone-beam computed tomography with a flat-panel imager: Initial performance characterization," Med. Phys., vol. 27, no. 6, pp. 1311-1323, 2000.

[4] H. K. Kim, S. C. Lee, M. H. Cho;, S. Y. Lee, and G. Cho, "Use of a flat-panel detector for microtomography: A feasibility study for smallanimal imaging," IEEE Trans. Nucl. Sci., vol. 52, no. 1, pp. 193-198, Feb. 2005 .

[5] I. Fujieda, G. Cho, J. Drewery, T. Gee, T. Jing, S. N. Kaplan, V. Perezmendez, D. Wildermuth, and R. A. Street, "X-Ray and charged-particle detection with Csi(Tl) layer coupled to a-Si-H photodiode layers," IEEE Trans. Nucl. Sci., vol. 38, no. 2, pp. 255-262, Feb. 1991.

[6] E. Miyata, M. Miki, N. Tawa, D. Kamiyama, and K. Miyaguchi, "Development of new X-ray imaging device sensitive to $0.1-100 \mathrm{keV}$," Nucl. Instrum.Meth. Phys. Res. A, vol. 525, no. 1-2, pp. 122-125, 2004.

[7] J. J. Vaquero, E. Lage, L. Ricon, M. Abella, E. Vicente, and M. Desco, "rPET detectors design and data processing," in Proc. IEEE NSS-MIC Conf. Record, 23-29, 2005, vol. 5, pp. 2885-2889.

[8] Y. Wang, J. Seidel, B. M. W. Tsui, J. J. Vaquero, and M. G. Pomper, "Performance evaluation of the GE healthcare eXplore VISTA dualring small-animal PET scanner," J. Nucl. Med., vol. 47, pp. 1891-1900, 2006.
[9] S. Redondo, J. J. Vaquero, E. Lage, M. Abella, G. Tapias, A. Udias, and M. Desco, "Assessment of a new CT system for small animals," in IEEE NSS-MIC Conference Record, San Diego, 2006.

[10] E. Van de Casteele, "Model-based approach for beam hardening correction and resolution measurements in microtomography," Faculty Wetenschappen, Department Natuurkunde Antwerpen University Antwerpen, 2004.

[11] F. Noo and R. Clackdoyle, "Analytic method based on identification of ellipse parameters for scanner calibration in cone beam tomography," Phys. Med. Biol., vol. 45, pp. 3489-3508, 2000.

[12] G. T. Gullberg, B. M. W. Tsui, C. R. Crawford, J. G. Ballard, and J. T. Hagius, "Estimation of geometrical parameters and collimator evaluation for cone-beam tomography," Med. Phys., vol. 17, pp. 264-272, 1990.

[13] J. Li, R. J. Jaszczak, H. Wang, K. L. Greer, and R. E. Coleman, "Determination of both mechanical and electronic shifts in cone-beam SPECT," Phys. Med. Biol., vol. 38, pp. 743-754, 1993.

[14] Hamamatsu Application Manual Mar. 2003, X-ray flat panel sensor C7912 \& C7942 \& C7943, rev 2.10, kr1-i50006.

[15] E. Lage, J. J. Vaquero, S. Redondo, M. Abella, G. Tapias, A. Udías, and M. Desco, "Design and development of a high performance micro-CT system for small-animal imaging," presented at the IEEE NSS-MIC Conference Record, San Diego, CA, 2006.

[16] L. A. Feldkamp, L. C. Davis, and J. W. Kress, "Practical cone-beam algorithm," J. Opt. Soc. Amer. A, vol. 1, pp. 612-619, 1984.

[17] S. M. Bentzen, "Evaluation of the spatial resolution of a CT scanner by direct analysis of the edge response function," Med. Phys., vol. 10, no. 5, pp. 578-580, 1983.

[18] U. Ewert, U. Zscherpel, and K. Bavendiek, "Replacement of film raduiography by digital techniques and enhancement of image quality," [Online]. Available: www.ndt.net/search/docs.php3?id=4516 2007

[19] American Society for Tests and Materials 2001, Standard Test Method for Measurement of Computed Tomography (CT) System Performance.

[20] H. H. Barrett, S. K. Gordon, and R. S. Hershel, "Statistical limitation in transaxial tomography," Comput. Biol. Med., vol. 6, pp. 307-323, 1976.

[21] N. L. Ford, M. M. Thornton, and D. W. Holdsword, "Fundamental image quality limits for microcomputed tomography in small animals," Med. Phys., vol. 30, no. 11, pp. 2869-2877, 2003.

[22] A. Obenaus and A. Smith, "Radiation dose in rodent tissues during micro-CT imaging," J. X-Ray Sci. Technol., vol. 12, pp. 241-249, 2004.

[23] M. J. Paulus, H. Sar-Sarraf, S. S. Gleason, M. Bobrek, J. S. Hicks, D K. Johnson, J. K. Behel, L. H. Thompson, and W. C. Allen, "A new X-ray computed tomography system for laboratory mouse imaging," IEEE Trans. Nucl. Sci., vol. 46, no. 3, pp. 558-564, Jun. 1999.

[24] W. Ross et al., "Design and performance characteristics of a digital flat-panel computed tomography," Med. Phys., vol. 33, no. 6, pp. 1888-1901, 2006. 